\title{
Tools for the Design of Multiphase Processing Operations
}

\author{
A.M. Al Taweel, F. Azizi, K. Podila, S. Madhavan, M. Koksal and Y. P. Gupta \\ Faculty of Engineering, Dalhousie University, Halifax, Nova Scotia B3J 2 X4 \\ Al.Taweel@Dal.Ca
}

\begin{abstract}
Although multiphase systems are encountered in a broad range of operations it is still very difficult to design such operations without recourse to a large degree of empiricism and know-how. This is mainly caused by the difficulty of properly simulating bubble/drop breakage and coalescence processes. The use of population balance equations and computational fluid dynamics offer an avenue for overcoming this obstacle. The ability to properly understand and quantify the complex phenomena taking place in multiphase systems was found to promote the innovation process which can enable the achievement of order-of-magnitude improvement in process performance.
\end{abstract}

\section{Introduction}

Fluid mechanics plays an essential role in the chemical process industries since the hydrodynamic conditions prevalent in any processing unit play a vital role in determining its performance efficiency. Design information concerning this aspect is traditionally obtained using experimental, semi-theoretical, and mathematical methods; a practice that conceals many of the hydrodynamic details and non-idealities. Consequently, many of the equipment designs presently in use are based on the experience of experts applying rules of thumb, an approach that resembles art more than science. Processes that are sensitive to local phenomena and reactant concentrations are therefore difficult to design or scale-up, because the design correlations do not take scaleup into account [1].

The widespread use of empirical correlations poses several limitations. For instance, results obtained from such correlations cannot be used over parameter ranges not included in the original measurement data set, totally ruling out the possibility of any extrapolated inferences. Additionally, the correlations used are presented in a very simplified form and are based on several assumptions rendering them inapplicable to many practical situations without the incorporation of excessive safety margins. As a means to overcome these limitations, the computational modeling approach was adopted.

Computational modeling is concerned with the formulation of real-world complex problems and developing solution methods to solve these problems. In the beginning, plain mathematical modeling (that involved simulations using numerical schemes worked out by hand) was used. This usually necessitated the use of overly simplistic assumptions concerning parameters such as flow patterns, heat and mass transfer rates, and the use of a uniform energy dissipation rate throughout the volume of interest. This later evolved into a situation where the volume of interest is divided into several interconnected compartments and the principles of mass, momentum and energy conservation applied to each compartment (Fig. 1). The outputs from one compartment are used as inputs for the succeeding compartment and so on; finally, the results are combined and used to obtain the overall picture. One of the benefits of such an approach is that the spatial variation of the various parameters can be taken into account. However, this approach is very cumbersome and is prone to excessive errors unless a very large number of compartments are used. These problems can be overcome by using Computational Fluid Dynamics [CFD] to model various processing operations. Computational fluid dynamics allows the analysis of fluid flow problems in detail, faster and earlier in the design cycle than is possible with experiments. Its use thus results in lowering the cost and risks involved in the design process. Solving a fluid flow problem typically involves discretizing the domain of interest into a finite set of elements (varying between 1,000 up to one million or more depending on the complexity of the problem) and applying the equations of conservation of mass, momentum, energy, and chemical species (where necessary) to these elements. Boundary conditions are placed at the edges of the domain of interest where initial conditions are specified for the flow parameters in the domain and the resultant algebraic equations are solved in an iterative fashion [2]. In the beginning, CFD codes 


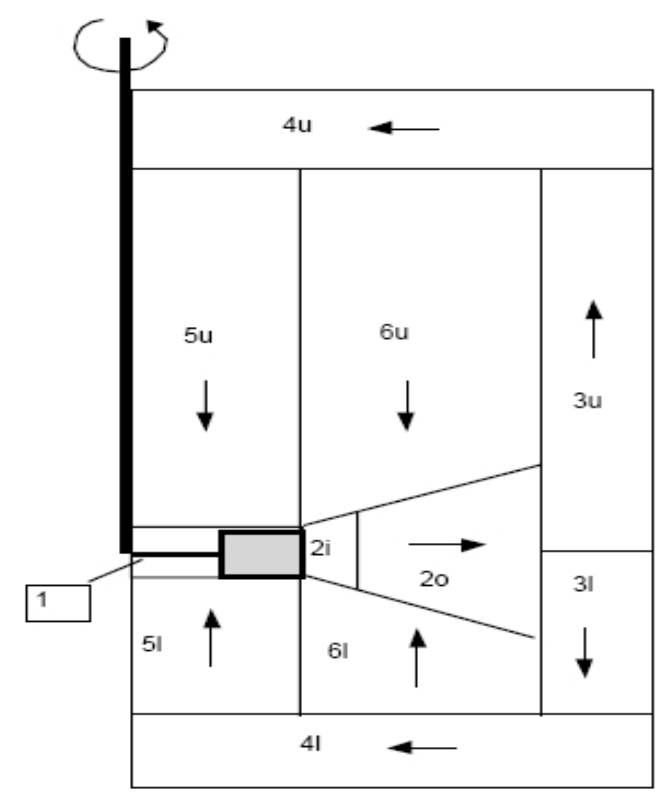

Figure 1. The multi-compartment approach to simulating complex flow fields [3]

were written for specific problems, but this practice was rapidly replaced by general-purpose CFD codes which became increasingly used in the design process as powerful computational resources became more readily available.

The defense and aerospace industries were the initial beneficiaries of CFD simulations because of their ability to access supercomputers at an early stage. However, the automotive and nuclear industries quickly followed suit and CFD is now being used in the design of a wide range of items such as artificial heart valves, racing boats, internal combustion engines, and drag reducing suits [4]. The complicated physics and chemistry involved in many process operations (including multiphase flows and chemical reactions) resulted in delaying the application of CFD in the Chemical Process Industries to a later date. It is presently used in many process applications such as:

- Combustion and Power generation [5]

- Flocculation operations [6]

- $\quad$ Spray drying of food ingredients [7]

- Tubular Chemical Reactors [8]

- Static Mixers and Hydrocyclones [9]

- Performance evaluation of electric vehicle batteries [10]

- Metal casting [11]

As can be seen from Fig. 2, CFD allows for the development of an interactive environment in which the designer can easily visualize and quantify the effect of various design and/or operating conditions on the performance of the processing unit (velocity field, temperature field, concentration field, turbulence quantities, heat and mass transfer rates, reaction rates, etc.).

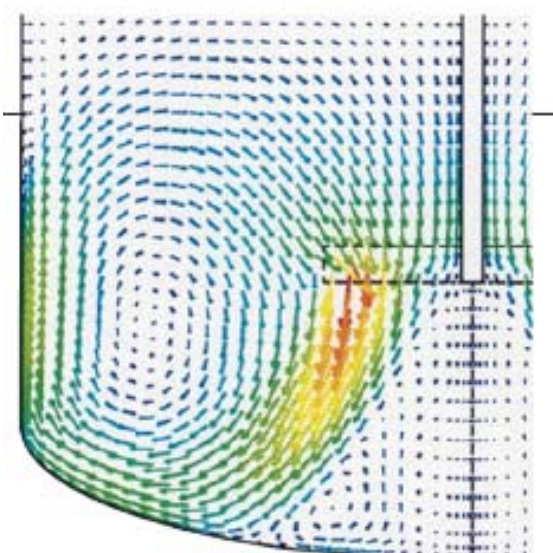

Detail of vector plot

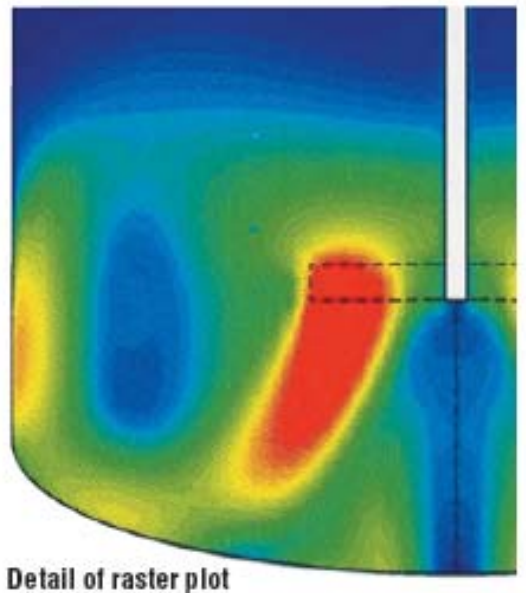

Figure 2. Velocity fields in a mechanically agitated tank (single phase) [12]

The use of CFD in the design of processing units results in significant reduction in the number of essential experiments that need to be conducted and offers the following advantages:

- CFD requires few restrictive assumptions and gives a complete description of the flow fields for all variables. Complex configurations can thus be treated and methods used are relatively easy to apply,

- CFD can quantitatively predict the flow, heat and mass transfer parameters in the domain of interest. Additionally, local fluid dynamic conditions can also be recorded for extensive analysis, 
- Modern CFD solutions are often quite comparable to, or even exceed the accuracy and resolution of the laboratory experiments. Using CFD, one can thus gain greater insight into the flow behavior without the complication and expense of setting up a range of experiments,

- Realistic models can be easily achieved. There is no need for scaling-down the models,

- Computational prediction costs are many orders of magnitude lower than complicated experimental investigation. This factor becomes more important as the physical situation under study becomes larger and more complex,

- The implications of many different configurations can be assessed in a shorter time frame than the experimental investigation. Virtual tests can be easily conducted leading to a clear perception of the problem in question.

Unfortunately, the situation is somewhat different in the case of multi-fluid systems (liquid-liquid and gasliquid systems) where it is necessary to incorporate population balance models [PBE] within the CFD framework in order to account for drop/bubble breakage and coalescence. The uncertainty surrounding such a modeling approach (particularly in the case of industrially relevant situations) restricts its applicability.

This paper discusses the factors affecting the accuracy and reliability of this design approach, and how it is affected by the modeling assumptions and numerical solution techniques. A case study is used to illustrate how PBE and CFD can be used to facilitate innovation at the design stage and accurately predict the effect of varying operating and design conditions on the technical and economic process performance.

\section{Multi-fluid Systems}

Multi-fluid systems are a sub-group of multiphase systems in which the dispersed phase is made up of bubbles and/or drops. These include liquid-in-gas, gasin-liquid as well as liquid-in-liquid dispersions which are encountered in a broad range of industries (such as the production, storage and transport of oil and gas resources, the chemical process industry, biotechnology, mineral and metal processing, energy conversion, separation technology, and environmental technology). Operations such as distillation, absorption, stripping, multi-phase reactions liquid extraction, direct contact heat transfer, biological wastewater treatment, ozonation, evaporation, steam condensation, spraying, and fuel atomization involve multi-fluid systems. The performance of these operations (e.g. conversion efficiency, heat and mass transfer rates) is dictated by the detailed structure of the multi-phase flow and the interaction between the phases.

These operations are difficult to simulate accurately because the dispersed phase entities (bubbles and/or drops) continuously undergo dispersion and coalescence processes. Generally speaking, the dispersion or breakage process can be defined as the reduction of the length scale of the dispersed phase bubbles or drops. It takes place under the action of external deforming forces (turbulent pressure fluctuations, viscous stresses due to velocity gradients) if they gain enough energy to compensate for the increase in surface energy associated with the formation of smaller bubbles/drops. Coalescence is the opposite of breakup process and results in the formation of larger bubbles/drops when two or more dispersed phase entities join together into one entity.

When two or more bubbles/drops collide under the action of laminar or turbulent shear, a small amount of liquid is trapped between them. The liquid film trapped between the adjacent bubble/drop surfaces then drains until the film becomes unstable and ruptures, leading to coalescence taking place. The relative magnitude of the coalescence and contact times are the most important parameters determining whether coalescence will take place or not. For instance, if the duration of contact between two colliding bubbles/drops (the contact time) is longer than the time taken for the drainage of the continuous phase film coalescence takes place. Film drainage is usually the rate limiting step in the coalescence process particularly in the presence of interfacial contaminants (a typical industrial situation). Very high levels of turbulence can thus result in violent collisions but insufficient time for film drainage [13].

The counteracting breakage and coalescence processes (Fig. 3) take place in every point throughout the contactor's volume with the net change in bubble/drop size distribution being a function of the relative magnitude of the two opposing processes. The dispersion will therefore tend to become finer in regions of high energy dissipation rates, but will tend to coalesce into a coarser dispersion as the bubbles/drop migrate to regions of lower energy dissipation rates. Dynamic equilibrium between the dispersion and coalescence processes (where no change in drop size and drop size distribution is observed) is only approached when the dispersion is exposed to uniform shear conditions for relatively long times. 


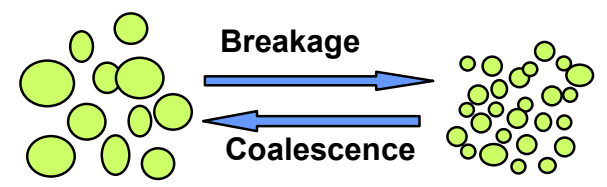

Figure 3. Dynamic equilibrium between bubble/drop breakage and coalescence processes

The breakage and coalescence of bubbles and drops is usually simulated using population balances, a concept that was introduced into the Chemical Process Industries in the early $60 \mathrm{~s}$ and found widespread applicability in describing particulate systems [14]. It can be considered as a particular statement of the equation of continuity when applied to the dispersed phase present in a control volume depicted in Fig. 4.

Net rates of the entities transportation through the boundaries of the control volume by convection are caused by velocity, concentration, temperature and pressure gradients and/or external forces (such as gravity and centrifugal forces) acting on the entities. Within the control volume boundaries depicted in Fig. 4, entities may also be generated or destroyed by coalescence, breakage, and chemical reaction. For large control volumes where the shear stresses encountered by the dispersed phase entities can vary from one location to the other, the number and volume of the dispersed phase entities can be a function of position and time [15]. This complication can however be eliminated by selecting control volumes that are small enough that the assumption of uniform hydrodynamic conditions (within the control volume) holds true.

All these phenomena combined, cause changes in the number density of entities within the control volume which are expressed by the population balance equation as follows:

Rate of accumulation of particles in the control volume $=$ Net rate of transport into the control volume by convection + Net rate of generation in control volume by breakage and coalescence + Net rate of generation in control volume by other means.

Since bubbles and drops are present in a wide range of sizes (Fig. 5), the population is divided into several sub-groups each having a narrower drop size range.

For bubbles and drops the net rate of generation by breakage and coalescence is equal to:

Birth rate by coalescence + Birth rate by breakage Death rate by coalescence - Death rate by breakage

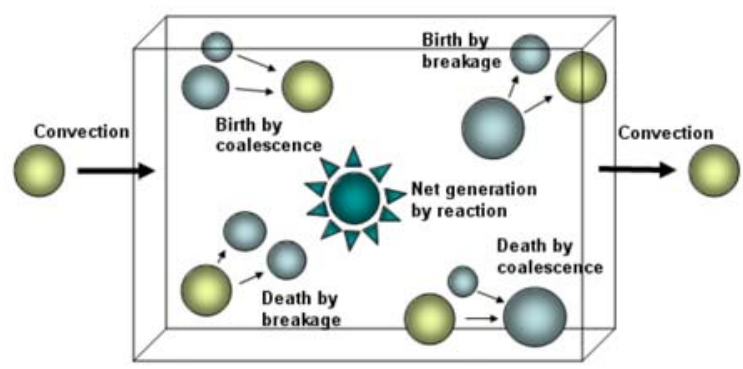

Figure 4. Control volume for PBE

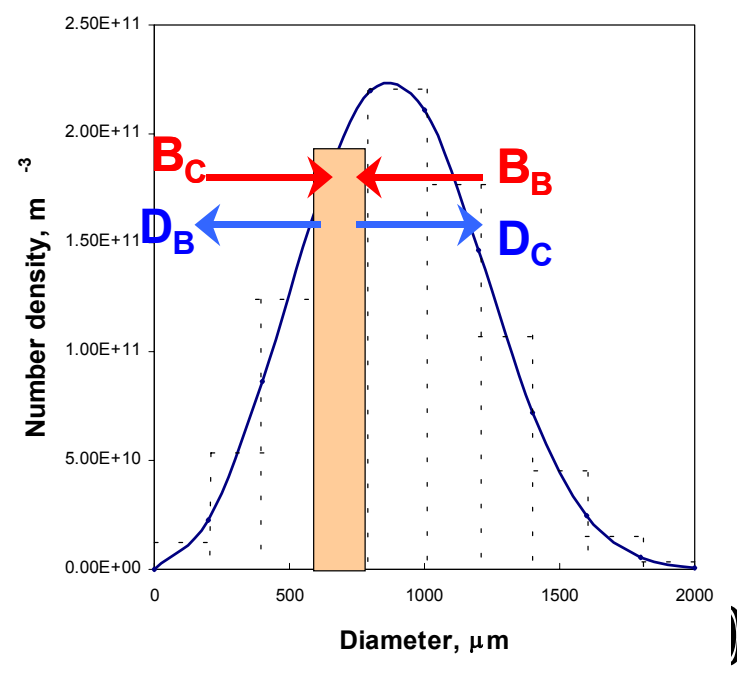

Figure 5. Graphical representation of drop birth/death by coalescence and breakage

However, use of population balance has not been enthusiastically adopted by industry because:

- The use of overly simplified hydrodynamic assumptions in most of the modeling work conducted prior to 1998 (e.g. assuming uniform energy dissipation rate in mechanically agitated tanks),

- The inability to discriminate amongst the various models proposed for bubble/drop breakage and coalescence sub-processes (collision frequency, coalescence efficiency, breakage frequency, number of daughter drops per breakage event, and daughter drop size distribution),

- The indiscriminate use of models was found to result in order-of-magnitude errors in estimating the breakage and coalescence rates [13],

- Uncertainty about the mathematical errors introduced while solving the PBE,

- The lack of experimental results obtained under known hydrodynamic conditions where the PBE assumptions hold. 
The first source of difficulty is eliminated when PBE are embedded within CFD codes (as has recently been the case for four commercial suppliers); although this significantly increases the computational demands needed to solve a particular solution.

\section{Liquid Dispersion in Static Mixers}

The operational characteristics of screen-type static mixers was investigated by using dilute liquid-liquid dispersions flowing in a $25.4 \mathrm{~mm}$ ID pipe loop [16]. The continuous and dispersed phases were introduced to a vertical $1.5 \mathrm{~m}$ long mixing section where dispersion is induced using a series of equally spaced screens. The characteristics of the screens used are given in Table 1.

Table 1. Characteristics of the woven screens investigated

\begin{tabular}{cccc}
\hline $\begin{array}{l}\text { Screen } \\
\text { No. }\end{array}$ & $\begin{array}{c}\text { Wire Size } \\
(\mathrm{mm})\end{array}$ & $\begin{array}{c}\text { Open Area } \\
(\%)\end{array}$ & $\begin{array}{c}\text { Mesh Size } \\
(\mathrm{mm})\end{array}$ \\
\hline 1 & 0.508 & 27 & 0.55 \\
2 & 0.15 & 33 & 0.21 \\
3 & 0.305 & 41 & 0.54 \\
\hline
\end{tabular}

The drop size distribution obtained at different design and operating conditions was recorded using a video camera with very short exposure times and the resulting images (Fig. 6) were analyzed using semiautomated image analysis software for measuring the sizes of the drops present in the dispersion.

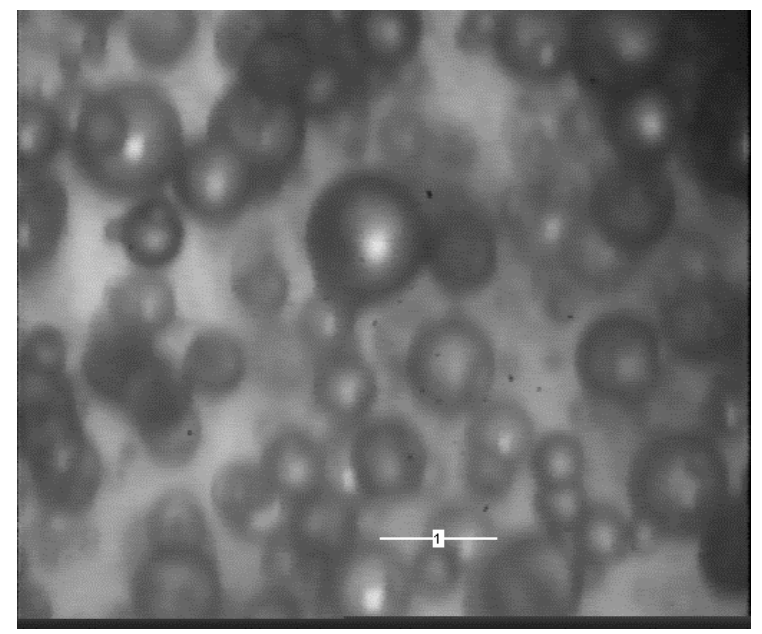

Figure 6. Typical image of the dispersion
The system investigated was a dispersion of Bayol Oil (a highly aliphatic light mineral oil supplied by ESSO with a density $792 \mathrm{~kg} / \mathrm{m}^{3}$, viscosity of $226 \mathrm{cP}$ at $20{ }^{\circ} \mathrm{C}$ and interfacial tension of $19.0 \mathrm{mN} / \mathrm{m}$ ) in tap water. Additional information concerning the experiments are given in [17].

The range of experimental conditions investigated can be summarized as,

$\begin{array}{ll}\text { Number of Screen elements } & 0 \text { to } 15 \\ \text { Inter-screen spacing } & 5 \text { and } 10 \mathrm{~mm} \\ \text { Superficial velocity, } U_{P}, & 0.3 \text { to } 1.94 \mathrm{~m} / \mathrm{s} \\ \text { Screen open area, } \alpha, & 27 \text { to } 41 \% \\ \text { Dispersed phase holdup, } \phi & 0.5 \text { to } 4 \% \\ \text { Pipe Weber number, We } \mathrm{P}, & 119 \text { to } 3010 \\ \text { Pipe Reynolds numbers } & 7,000 \text { to } 50,000 \\ \text { Jet Weber numbers } & 713 \text { to } 41,285 \\ \text { Mean energy dissipation rate } & 5.6 \text { to } 1,316 \mathrm{~W} / \mathrm{Kg}\end{array}$

The use of screen-type static mixers generates a uniform flow fields across the pipe's cross section and the turbulence intensity in the regions adjacent to the screens was varied by changing the superficial velocity and screen characteristics [18]. Although the turbulence field is uniform in the radial direction, it decays rapidly in the downstream direction and turbulence essentially decays within a distance of 2-5 mm (Fig. 7). The use of multi-stage screen-type static mixers provides the following hydrodynamic characteristics which are necessary for the use of PBE,

- Narrow residence time distributions (plug flow).

- Uniform, and well known, hydrodynamic conditions for the flowing gas and liquid phases.

- Minimum spatial variation of the local energy dissipation rate across the flow direction.

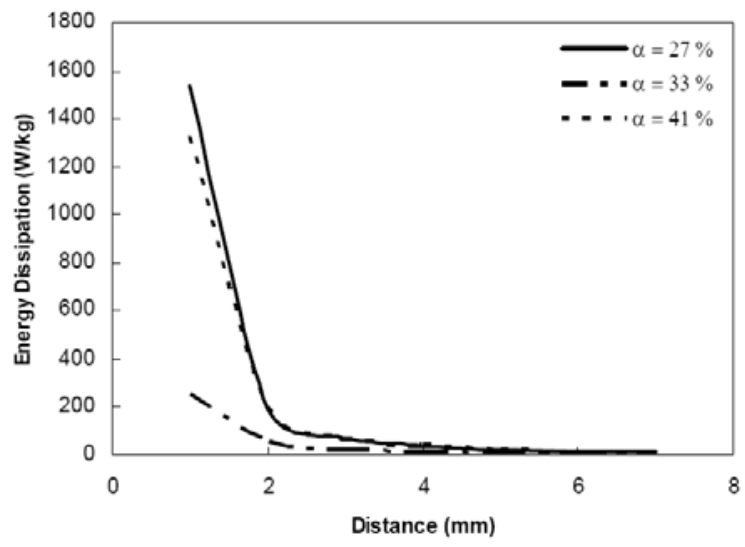

Figure 7. Effect of screen open area on the rate of energy dissipation $(U=1.94 \mathrm{~m} / \mathrm{s})$ 


\section{Simulating the Dispersive Performance of the Static Mixer}

The experimental data thus obtained cover a wide range of design and operating conditions present an excellent opportunity to test the suitability of using PBE to simulate bubble/drop dispersion and coalescence processes. Contrary to most previous data used for such purposes (most of which were obtained using MAT where highly non-uniform hydrodynamic conditions are encountered) these data were obtained under radially uniform turbulence conditions that approach the ideal situation of isotropic turbulence. To facilitate comparison over a wide range of experimental conditions, and increase the sensitivity of fit to variations in model parameters, the experimental distributions were normalized with respect to the Sauter mean diameter and used as a basis for the comparison.

Drop breakage and coalescence rates were described using two different models:

- The model developed by Rod and Misek [19] after modifying it in order to account for variation in the value of the local energy dissipation rate.

- The model developed by Coulaloglou and Tavlarides [20].

The value of the adjustable parameters (two for the Rod and Misek model and five for the Coulaloglou and Tavlarides) were adjusted in order to force model predictions fit the drop size distribution obtained under the well developed turbulent dispersion conditions observed at a superficial velocity of $0.97 \mathrm{~m} / \mathrm{s}$. As can be seen from Fig. 8, the models were found to be capable of fitting the experimental results quite well. However, the fit constants were found to be significantly different from those reported in literature. This discrepancy is most probably due to the simplifying assumptions used by many authors who validated their PBE using data obtained using Mechanically Agitated Tanks (assuming a uniform local energy dissipation rate throughout the mixer volume). This clearly illustrates the danger of using overly simplified hydrodynamic assumptions in PBE modeling and the errors that can be introduced by using the breakage and coalescence models under conditions where they do not truly apply. For example, the drop size distributions obtained using the classical values were found to be an order of magnitude larger than those observed in the present investigation.

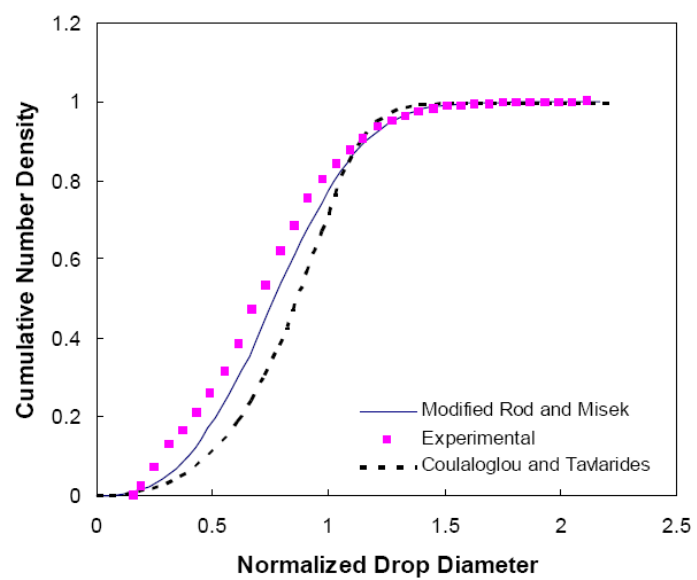

Figure. 8. Cumulative Drop number density distribution $(\mathrm{f}=0.5 \% ; U=0.97 \mathrm{~m} / \mathrm{s} ; \mathrm{a}=$ $33 \%)$

To validate the suitability of the fit parameters obtained by this approach, they were used to predict the effect of various operating and design parameters (superficial velocity, percentage open area of the screen) on the resulting drop size distribution. Very good agreement with the experimental results was obtained except at very low velocities where a second breakage mechanism takes over.

\section{Using PBE to Optimize the Design of Liquid-Liquid Contactors}

The afore mentioned ability to predict the effect of various operating and design parameters on the liquidliquid dispersion generated by static mixers was taken advantage of to identify the optimum design and operating conditions for a fuel desulphurization unit in which a similar contactor was used [17].

The Imperial Oil desulphurization approach focuses on removing elemental sulfur and is based on the use of the inverse Doctor Treatment in which the sulfurcontaining fuels are contacted with an aqueous caustic solution of sodium sulfide $\mathrm{Na}_{2} \mathrm{~S}$. The elemental sulfur is converted to water soluble polysulfides according to the following reaction.

$$
\mathrm{Na}_{2} \mathrm{~S}+3 \mathrm{~S} \rightarrow \mathrm{Na}_{2} \mathrm{~S}_{4}
$$

This reactive liquid-liquid extraction operation is usually conducted in a multi-stage mechanically agitated column equipped with Rushton-type impellers. At medium and elevated temperatures, the reaction rates are expected to be high and the overall reaction becomes controlled by the inter-phase rate of mass transfer which, in turn, is strongly affected by the drop size distribution generated by the mixer. At the typical reaction temperature of $50^{\circ} \mathrm{C}$, a contact time of 
12.5 minutes was reportedly needed in order to reduce the sulfur contents from $26.4 \mathrm{ppm}$ down to $1.8 \mathrm{ppm}$ when a MAT is used under batch operation. On the other hand, the preliminary results obtained when screen type static mixers were used for this purpose indicate that the following benefits can be accrued [16],

- The residence time needed to reduce the residual colloidal sulphur from 24 to $1.7 \mathrm{ppm}$ can be reduced by a factor of 25 ,

- The treatment temperature can be reduced from $50^{\circ} \mathrm{C}$ down to $29^{\circ} \mathrm{C}$ without adversely affecting the reaction rate,

- The specific power consumption was reduced from around 0.6 down to $0.08 \mathrm{kWh}$ per tonne of diesel treated.

These data indicate that volumetric mass transfer coefficients as high as $0.1 \mathrm{~s}^{-1}$ can be easily achieved by using screen-type static mixers, a value which is significantly higher than those reported for most other contactors. A large part of this improved performance can be attributed to the large interfacial areas in the regions adjacent to the screens and the ability of micromixing to improve inter-phase mass transfer. The ability to achieve high interfacial areas of contact between the phases, a, is thus beneficial because it enhances the value of the volumetric mass transfer coefficient ( $\mathrm{k} \mathrm{a}$ ). Furthermore, in accordance with the Frössling equation $\left(\mathrm{k}=2 \mathrm{D} / \mathrm{d}_{32}\right.$ for contaminated surfaces with no slip between the phases), the interphase mass transfer coefficient, $\mathrm{k}$, is expected to increase as the drop sizes decrease.

In order to optimize the design of this innovative contactor, the effect of various operating and design conditions on the inter-phase rate of mass transfer achievable in a screen-type static mixer was simulated using PBE (to calculate a and $\mathrm{d}_{32}$ ) in combination with the Frössling equation (to calculate $\mathrm{k}$ ). A typical result is depicted in Fig. 9 which clearly shows that the dispersion becomes progressively finer as the diesel/caustic solution passes through successive screens. The local mass transfer coefficient increases every time the two-phase mixture passes through a screen (due to the shifting of the dynamic equilibrium towards the formation of finer drops) with a maximum being observed in the downstream regions adjacent to the screen (i.e. the region of very high local energy dissipation rate, Fig. 7). However, drops tend to coalesce as they move into regions of lower energy dissipation rates; consequently, the mass transfer coefficient progressively decreases as the stream moves further away from the screen.

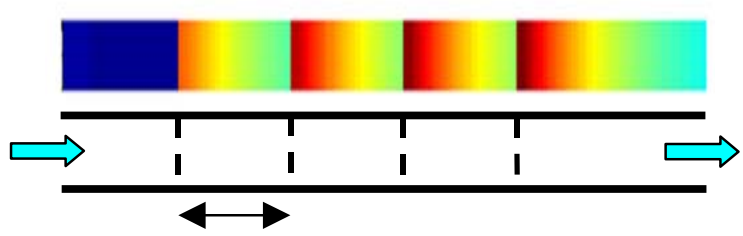

Figure 9. Inter-phase mass transfer coefficient in screen-type static mixers

The absolute value of the mass transfer coefficient that can be achieved is thus controlled by the superficial velocity through the pipe and the percentage open area in the screen. On the other hand, the inter-screen spacing controls the average value within a stage and the time of contact between the phases. As can be seen from Fig. 10, very high average mass transfer coefficients (which leads to smaller and les costlier reactors) can be achieved by using short inter-screen spacing. This comes at the expense of larger energy consumption rates (which lead to larger operating costs). On the other hand, the most energy effective operation is achieved at larger inter-screen spacing. The balance between those two desirable features is dictated by the specific situation at hand, availability of floor space, safety concerns etc. The predicted volumetric mass transfer coefficients compared reasonably well with the preliminary desulphurization results.

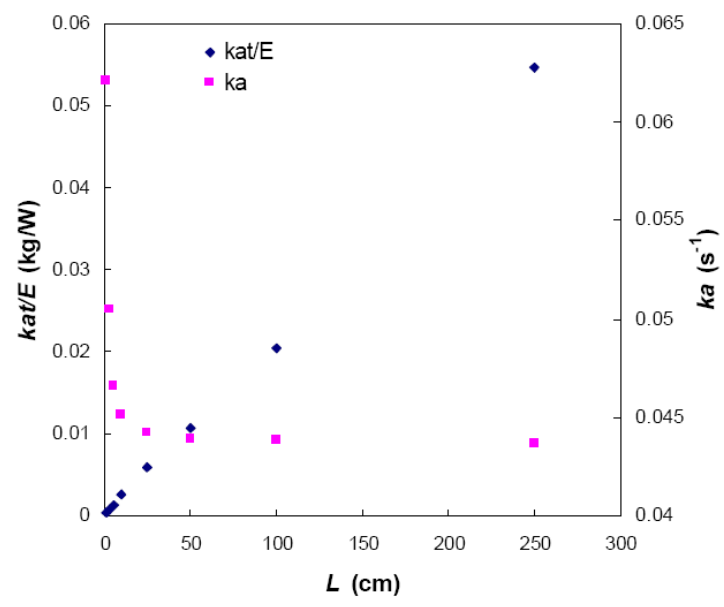

Figure 10. Effect of inter-screen spacing on the volumetric mass transfer coefficient and the mass transferred per unit power consumption $(U=1 \mathrm{~m} / \mathrm{s})$

Compared to conventional liquid -liquid contactor (MAT), the use of in-line static mixing elements offers the following benefits:

- Mechanical stability and absence of moving parts,

- Available in a wide variety of construction materials, 
- Very low maintenance requirements,

- Short and narrow residence time distribution,

- Large interfacial area of contact can be achieved with minimal risk of phase-inversion or the formation of difficult-to-separate haze,

- The high micro-mixing rates encountered in the regions behind screens are expected to enhance inter-phase mass transfer,

- Favorable concentration driving force (plug flow vs. CSTR),

- Improved conversion efficiency and process yield (better quality control and lower operating cost in the subsequent separation step),

- The combined effect of the above-mentioned factors results in significant reduction in the contactor's volume,

- A major reduction of capital cost requirements (equipment, support structure, foundations),

- Suitability for limited spaces and easiness of incorporation in existing processes (pipelines become the contacting device),

- Significantly lower operating cost,

- Improved intrinsic safety (smaller in-plant inventories of hazardous materials).

\section{Conclusions}

Computational fluid dynamics [CFD] is a numerical tool that is increasingly being used to design a wide range of engineering fields that rely on a good understanding of interaction with fluids (e.g. automotive and aerospace industries, fluid pumping and distribution, combustion systems). However, it needs to incorporate population balance models (that accounts for drop/bubble breakage and coalescence) in order to handle multi-fluid systems.

This paper discusses the factors affecting the accuracy and reliability of this design approach, and how it is affected by the modeling assumptions and numerical solution techniques. A case study is used to illustrate how population-balance-equations and computationalfluid-dynamics can be used to accurately predict the effect of varying operating and design conditions on process performance. The insight gained by using such tools was essential for the development of a novel multiphase contactor that could reduce the size of a fuel desulphurization unit.

\section{References}

[1] A.Bakker, A. H. Haidari and L.M. Oshinowo, "Realize greater benefits from CFD", Chemical Engineering Progress, American Institute of Chemical Engineers, New York, 2001, pp. 45-53.
[2] Blazek, J., "Computational Fluid Dynamics: Principles and Applications", Elsevier, Balden-Daettwil, Switzerland, 2001.

[3] V.Alopaeus, J. Koskinen and K. I. Keskinen, "Simulation of the population balances for liquid-liquid systems in a nonideal stirred tank. Part 1 Description and qualitative validation of the model", Chemical Engineering Science, Pergamon Press, Great Britain, 1999, 5887-5899.

[4] Fluent News, "Applied Computational Fluid Dynamics” Vol. XIII Issue 1, Spring 2004.

[5] P.J. Stopford,"Recent applications of CFD modelling in the power generation and combustion industries", Applied Mathematical Modelling, Elsevier, New York, 2002,pp. 351374.

[6] J.J Ducoste and M.M. Clark, "Turbulence in Flocculators: Comparison of Measurements and CFD Simulations", AIChE Journal, American Institute of Chemical Engineers, New York, 1999, pp.432-436

[7] T.A.G. Langrish, and D.F. Fletcher, "Spray drying of food ingredients and applications of CFD in spray drying", Chemical Engineering and Processing, Pergamon Press, Great Britain, 2001, pp. 345-354.

[8] L.K Hjertager., B.H. Hjertager, and T. Solberg, CFD modelling of fast chemical reactions in turbulent liquid flows", Computers and Chemical Engineering, Pergamon Press, Great Britain, 2002, pp. 507-515.

[9] M. Casey, E. Lang, R. Mack, R. Schelgel and M. Wehrli, "Application of Computational Fluid Dynamics for Process Engineering at Sulzer" $23^{\text {rd }}$ SPEEDUP Workshop on Process Engineering, Switzerland, March 1998.

[10] W.B. Gu, C.Y. Wang and B.Y. Liaw, "The use of computersimulation in the evaluation of electric vehicle batteries",Journal of Power Sources, Elsevier, New York, 1998, 151-161.

[11] S.T. Johansen, "Multiphase flow modeling of metallurgical flows", Experimental Thermal and Fluid Science, Elsevier, New York, 2002, 739-745.

[12] D.S Dickey and J.B. Fasano, "How Geometry and Viscosity Influence Mixing", Chemical Engineering, Chemical Week Publishing, New York, 2004, pp 42-46.

[13] G. Sirijeerachai and A.M. Al Taweel, "Comparative Analysis of Drop Coalescence Models" $52^{\text {nd }}$ Canadian Chemical Engineering Conf., Vancouver BC Oct. 2002.

[14] Ramkrishna, D., Population Balances Academic Press, San Diego, 2000.

[15] G. Sirijeerachai, "Population balance analysis of MultiFluid Systems" Ph.D thesis, Dalhousie University, Canada, 2003. 
[16] M. El-Ali, "Performance Characteristics of a Novel Liquid-Liquid Contactor" Ph.D thesis, Dalhousie University, Canada, 2001.

[17] A.M. Al Taweel, M. El-Ali, F. Azizi, H.G. Gomaa, B. Liekens, D. Odedra and A. Uppal, "In-Line Processing for Intensifying Multi-Phase Contacting Operations" Proc. $5^{\text {th }}$ International Process Intensification Conference, BHR, Cranfield UK, 59-73 (2003).

[18] A.M. Al Taweel and C. Chen, "A Novel Static Mixer for the Effective Dispersion of Immiscible Liquids", Trans. Inst. Chem. E., Institution of Chemical Engineers, United Kingdom,1996, 445- 450.

[19] V. Rod and T. Misek, "Stochastic Modeling of

Dispersion Formation in Agitated Liquid-Liquid Systems", Trans. Inst. Chem. E., Institution of Chemical Engineers, United Kingdom, 1982, pp.48-53.

[20] C.A. Coulaloglou and L.L. Tavlarides, "Description of interaction processes in agitated liquid-liquid dispersions", Chemical Engineering Science, Pergamon Press, Great Britain, 1977, pp. 1289-1297. 